\title{
Superficial pseudoaneurysms: clinicopathologic aspects and involvement of extracellular matrix proteoglycans
}

\author{
Allen P Burke ${ }^{1}$, Hannu Jarvelainen², Frank D Kolodgie ${ }^{1}$, Ashita Goel ${ }^{1}$, Thomas N Wight ${ }^{2}$ \\ and Renu Virmani ${ }^{1}$ \\ ${ }^{1}$ Department of Cardiovascular Pathology, Armed Forces Institute of Pathology, Washington, DC, USA and \\ ${ }^{2}$ Department of Vascular Biology, The Hope Heart Institute, Seattle, WA, USA
}

\begin{abstract}
The distribution of proteoglycans in 21 temporal and two ulnar artery pseudoaneurysms was studied immunohistochemically. A history of trauma was elicited in six cases, and 16 of the lesions were pulsatile. The clinical diagnosis was aneurysm or cyst in $\mathbf{1 8}$ patients, possible arteritis in two patients, tumor in one patient, and unknown in the remaining patient. Histologically, there was a prominent myxoid neointimal response in the walls of each interrupted artery. The remnant arterial segment was often inconspicuous. Prominent smooth muscle cell proliferation and granulation tissue response with inflammation led to misdiagnosis of tumor or vasculitis, respectively, in 11 cases. Immunohistochemical staining for proteoglycans demonstrated abundant, diffuse versican in interrupted wall segments. Biglycan was confined to collagenized and vascularized areas. In some portions of medial disruption, in which angiogenesis was prominent, decorin was expressed within endothelial cells of neocapillaries. These findings demonstrate that superficial pseudoaneurysms may be mistaken clinically and pathologically for unrelated entities. The immunohistochemical studies confirm that versican is upregulated in areas of tensile stress. In addition, the presence of endothelial expression of decorin supports the concept of decorin's involvement in angiogenesis.
\end{abstract}

Modern Pathology (2004) 17, 482-488, advance online publication, 20 February 2004; doi:10.1038/modpathol.3800060

Keywords: pseudoaneurysm; proteoglycans; angiogenesis; decorin; versican

Pseudoaneurysms are a potential complication of arterial injury, especially trauma. Peripheral vessels such as the superficial temporal artery are particularly vulnerable to pseudoaneurysm formation because these vessels lack protection from the surrounding soft tissues. Vessel wall disruption results in a hematoma that organizes, encasing the damaged portion of the artery. With continued organization, blood flow through the artery may eventually return. ${ }^{1}$ Although computed tomography (CT) and angiography can be helpful in the diagnosis of pseudoaneurysms, superficial aneurysms are typically removed by simple surgical excision without an extensive preoperative evaluation. ${ }^{1-11}$

The pulsatile characteristics pointing to the diagnosis may be minimal or absent. ${ }^{12}$ However,

Correspondence: Dr R Virmani, MD, Department of Cardiovascular Pathology, Armed Forces Institute of Pathology, Washington, DC 20306-6000, USA.

E-mail: virmani@afip.osd.mil

Received 18 July 2003; revised 7 November 2003; accepted 11 November 2003; published online 20 February 2004 the vascular repair process in areas of increased tensile strength gives rise to the unique histologic features of pseudoaneurysms. Specifically, proteoglycans are known to accumulate in the regions of vascular lesions that are characterized by smooth muscle cell proliferation and migration. ${ }^{13-15}$ In addition, proteoglycan concentration varies with mechanical load, ${ }^{16}$ and synthesis of versican is increased when arterial smooth muscle cells are subjected to mechanical strain. ${ }^{17}$

The purpose of this study is two-fold. First, we are presenting the clinicopathologic features of a series of these lesions, because the histologic features are not often appreciated by practicing pathologists. On a more basic level, our other aim is to determine the distribution of proteoglycans within pseudoaneurysms in order to better understand the role of proteoglycans in the arterial repair.

\section{Materials and methods}

In all, 21 superficial temporal artery and two ulnar artery pseudoaneurysms were collected consecutively 
in consultation. Lesions were included if there was a mass lesion involving a peripheral muscular artery with discrete disruption of the media and internal elastic lamina, and associated chronic thrombus formation. Cases were excluded if remnant arteries showed histopathologic changes of inflammation or medial disease. The clinical history was reviewed from the pathology record for each case, specifically noting any history of associated trauma and presenting symptoms.

Elastic stains (Movat pentachrome) were performed at the time of initial consultation. Nine cases were selected retrospectively for immunohistochemical stains for proteoglycans (versican, biglycan, and decorin), ${ }^{18}$ and an additional seven for smooth muscle actin, calponin, smoothelin, and Ki-67. Before staining with proteoglycan antibodies, the sections were digested with chondroitin ABC lyase (ICN Biomedicals, Costa Mesa, CA, USA) at $250 \mathrm{mU} / \mathrm{ml}$ in $0.1 \mathrm{~mol} / \mathrm{l}$ Tris/acetate, $\mathrm{pH} 7.3$, for $1 \mathrm{~h}$ at $37^{\circ} \mathrm{C}$. Rabbit polyclonal antisera for the core proteins (aminoterminal peptides) of human biglycan (LF-51) and decorin (LF-136) were generously provided by Larry Fisher of the Craniofacial and Skeletal Disease Branch (National Institutes of Health, Bethesda, MD, USA). LF-51 was used at a 1:500 dilution. Rabbit antibody specific for the poly$\mathrm{E}$ region of human versican, VC-E, was kindly provided by Richard LeBaron (University of Texas at San Antonio, San Antonio, TX, USA) and was used at a 1:500 dilution. Control sections were incubated with the proper dilution of normal serum or IgG to match the conditions or isotype of the specific antibodies. Smooth muscle maturation markers included calponin (Dako, Carpinteria, CA, USA, 1:200 dilution), actin (muscle- $\alpha$-actin 1A4, Sigma, St Louis, MO, USA, dilution 1:4000), and smoothelin (Monosan, Uden, The Netherlands, 1:10 dilution). To identify proliferating cells, tissue sections were stained with the monoclonal MIB-1 antibody (Ki-67, ImmunoTech, Westbrook, ME, USA, 1:500 dilution). Positive control tissue consisted of normal human tonsil for Ki-67 and pig coronary arteries for smooth muscle markers; negative controls consisted of omission of the primary antibody. Immunostaining against Ki-67 nuclear antigen in paraffin sections was optimized by antigen retrieval. All reactions were visualized after incubating the sections with 1:500 dilution of streptavidin conjugated to horseradish peroxidase for $10 \mathrm{~min}$ after incubation in a matched secondary antibody. The color was developed with diaminobenzidine and contrast enhanced with $\mathrm{NiCl}_{2}$ (black reaction product); the sections were counterstained with methylgreen. Percent Ki-67 positivity was determined by histomorphometry.

\section{Results}

\section{Patient Characteristics}

The patient information is given in Table 1. There were 18 male and five female patients. The mean age was 35 years, \pm 19 years s.d. There was a history of prior trauma in six cases (three within 1 week, one after 1 day, one 2 years prior, and one had a history of frequent fistfights). In a seventh patient, there was a scar in the region of the aneurysm, but the patient

Table 1

\begin{tabular}{|c|c|c|c|c|c|c|}
\hline Case no. & Side & Age (years) & Sex & Pulsatile & Clinical diagnosis & Initial pathologic diagnosis \\
\hline 1 & Right $^{\mathrm{a}}$ & 15 & $\mathrm{M}$ & Pulsatile & Aneurysm & Other/unknown \\
\hline 2 & Right $^{\mathrm{a}}$ & 38 & $\mathrm{M}$ & Pulsatile & Aneurysm & Benign tumor \\
\hline 3 & Left & 6 & $\mathrm{M}$ & Nonpulsatile & Tumor & Sarcoma \\
\hline 4 & Right & 15 & $\mathrm{M}$ & Pulsatile & Aneurysm & Arteritis \\
\hline 5 & Bilateral ${ }^{\mathrm{b}}$ & 16 & $\mathrm{M}$ & Pulsatile & Aneurysm & Other/unknown \\
\hline 6 & Not stated & 18 & $\mathrm{M}$ & Nonpulsatile & Cyst & Benign tumor \\
\hline 7 & Left & 19 & M & Pulsatile & Cyst & Organized thrombus \\
\hline 8 & Left & 20 & $\mathrm{M}$ & Nonpulsatile & Cyst & Organized thrombus \\
\hline 9 & Right & 21 & $\mathrm{M}$ & Pulsatile & Pseudoaneurysm & Arteriovenous hemangioma \\
\hline 10 & Left & 22 & M & Nonpulsatile & Cyst & Pseudoaneurysm \\
\hline 11 & Right & 29 & $\mathrm{M}$ & Pulsatile & Aneurysm & Benign tumor \\
\hline 12 & Left & 29 & M & Pulsatile & Aneurysm & Inflammatory aneurysm \\
\hline 13 & Bilateral & 33 & $\mathrm{M}$ & Pulsatile & Aneurysm & Pseudoaneurysm \\
\hline 14 & Left & 36 & M & Nonpulsatile & Cyst & Benign tumor \\
\hline 15 & Bilateral & 37 & M & Pulsatile & Aneurysm & Organized thrombus \\
\hline 16 & Left & 40 & M & Pulsatile & Aneurysm & Arteritis \\
\hline 17 & Left & 46 & $\mathrm{~F}$ & Pulsatile & Aneurysm & Other/unknown \\
\hline 18 & Left & 47 & $\mathrm{~F}$ & Nonpulsatile & $\mathrm{R} / \mathrm{o}$ arteritis & Pseudoaneurysm \\
\hline 19 & Left & 56 & $\mathrm{M}$ & Pulsatile & Unknown & Arteritis \\
\hline 20 & Right & 61 & $\mathrm{~F}$ & Pulsatile & Aneurysm & Arteritis \\
\hline 21 & Right & 62 & $\mathrm{M}$ & Pulsatile & Cyst & Arteritis \\
\hline 22 & Left & 65 & $\mathrm{~F}$ & Nonpulsatile & $\mathrm{R} / \mathrm{o}$ arteritis & Other/unknown \\
\hline 23 & Left & 76 & $\mathrm{~F}$ & Pulsatile & Aneurysm & Benign tumor \\
\hline
\end{tabular}

${ }^{\mathrm{a}} \mathrm{Ulnar}$ artery; the remainder are temporal.

${ }^{\mathrm{b}}$ Contralateral aneurysm excised 3 years prior. 
denied knowledge of its cause. The duration of the lesion was, per patient history, $<1$ week in six cases, 1 week to 2 months in four cases, more than 2 months in eight cases, and unknown in five cases. In 16 cases, the mass was pulsatile; a clinical diagnosis of aneurysm was suspected in the majority of these patients. Three patients had bilateral lesions, two at the time of presentation, and one excised 3 years prior. Imaging studies including CT were performed in three patients, and demonstrated localized vascular lesions with normal brain and skull. In one, the CT scan showed a bony 'divot' underlying the lesion. There was no history of connective tissue disease in any patient, although one was described as 'marfanoid,' and in one the clinical diagnosis of Ehlers-Danlos syndrome was considered, but excluded by biochemical testing. In all patients, the simple excision was curative.

\section{Pathologic Findings}

The initial histopathologic impression was arteritis in six cases, pseudoaneurysm in three cases, organized hematoma or thrombus in three, benign neoplasm or arteriovenous hemangioma in six, sarcoma in one, and not stated in the remaining four. Histologically, the lesion was composed of organizing thrombus at the center of the lesion (Figure 1a), a remnant portion of media with abrupt borders (Figure 1b), and granulation tissue with abundant capillaries near the defect (Figure 1c), which was rich in proteoglycans (Figure 1d). Papillary endothelial hyperplasia was present in four, and prominent in the one case with a history of trauma the day before excision. Mitotic figures were present in the neovascular areas in 12 cases. In 16 cases, the remnant vessel with the interrupted media was identified initially; in seven, the remnant artery was seen only with recuts of the block. There were 'skip areas' separating breaks in the internal elastic lamina in seven cases; in 16, only one discrete interruption was identified. Two of the cases with skip areas had a history of trauma. The media remnant was unremarkable in every case except for the interrupted edge or edges. 'Epithelioid' or 'histiocytoid' change in the endothelial cells was present to some degree in all cases, but marked in four (Figure 2a and b). Eosinophils were present in five cases, including the four with marked epithelioid change (Figure 2a). There was no difference in the histologic findings (remnant artery, degree of epithelioid change, presence of eosinophils, degree of myxoid change) between cases with known history of trauma and those without.

\section{Immunohistochemical Findings}

Smooth muscle actin was present in all cases in the spindle cell area, and factor VIII-related antigendecorated endothelial cells surrounding the orga-

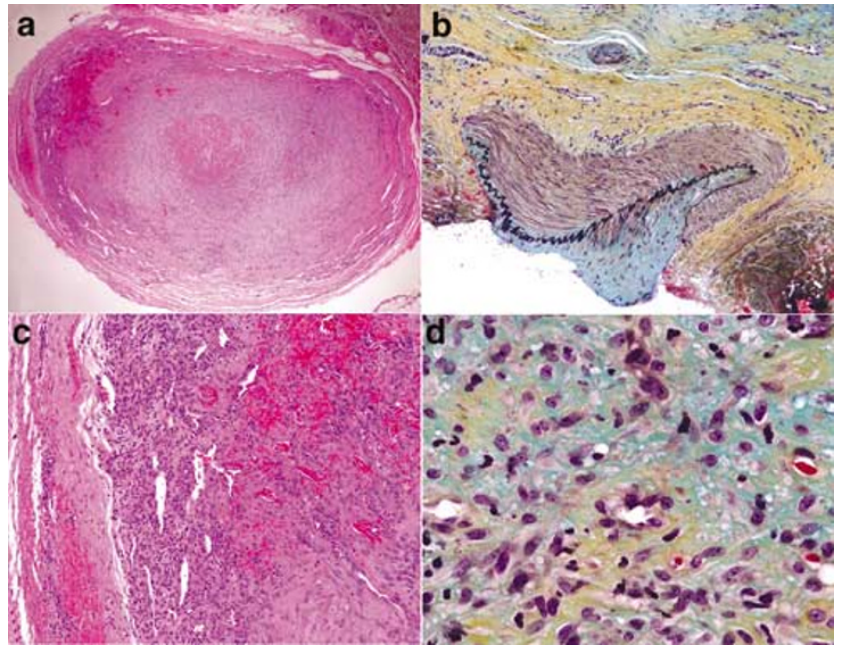

Figure 1 Arterial pseudoaneurysm (Case 6). (a) On low magnification stained by hematoxylin-eosin, there is a circumscribed mass with central organizing thrombus. (b) A higher magnification of the edge of the lesion at a different level stained for elastic (Movat pentachrome) demonstrates the remnant media. In this case, the remnant was difficult to identify, and multiple levels through the block were needed. (c) At the edge of the lesion there is marked neovascularity. (d) 'Epithelioid' endothelial cells are often present (Movat pentachrome). The patient was an 18-yearold man with a 'sebaceous cyst' on his temple for 1-2 months.

nized thrombus and in areas of epithelioid change. Decorin immunostaining was confined to microvascular CD31-positive endothelial cells frequently found in abundance at the base of the pseudoaneurysm and in areas of epithelioid endothelial cells (Figure 2c). Decorin was expressed in small neovessels in clusters of granulation tissue (Figure 3a), at the luminal surface (Figure $3 \mathrm{~b}$ ) and colocalized with factor VIII-related antigen expression (Figure 3c). Decorin occasionally was found in neovessels near the interrupted area of the internal elastic lamina (Figure 4a-d). Versican was found in all areas of the pseudoaneurysm wall, in a diffuse staining pattern (Figure 5). Biglycan staining was localized preferentially to outer areas of the organized pseudoaneurysm wall, in the areas of collagen deposition (Figure 6). Spindle cells in the proteoglycan matrix underlying the thrombus were strongly positive for smooth muscle actin in all cases. A subset of these cells expressed calponin (Figure 7a). Ki-67 staining demonstrated numerous positive cells in the smooth muscle cell proliferation, most numerous in the cases with a recent history of trauma. The mean \% proliferative index by Ki-67 staining was $17 \pm 4 \%$ in the most acute cases ( $<1$ week duration), $14 \pm 1.3 \%$ in the subacute cases (1 week-2 months), and $4 \pm 3 \%$ in the chronic cases ( $>2$ months). The difference between $<2$ and $>2$ months was significant $(P=0.02)$. These proliferating cells, which were smooth muscle actin positive as determined by examining serial cuts, were most numerous in the center of the proteoglycan-rich 

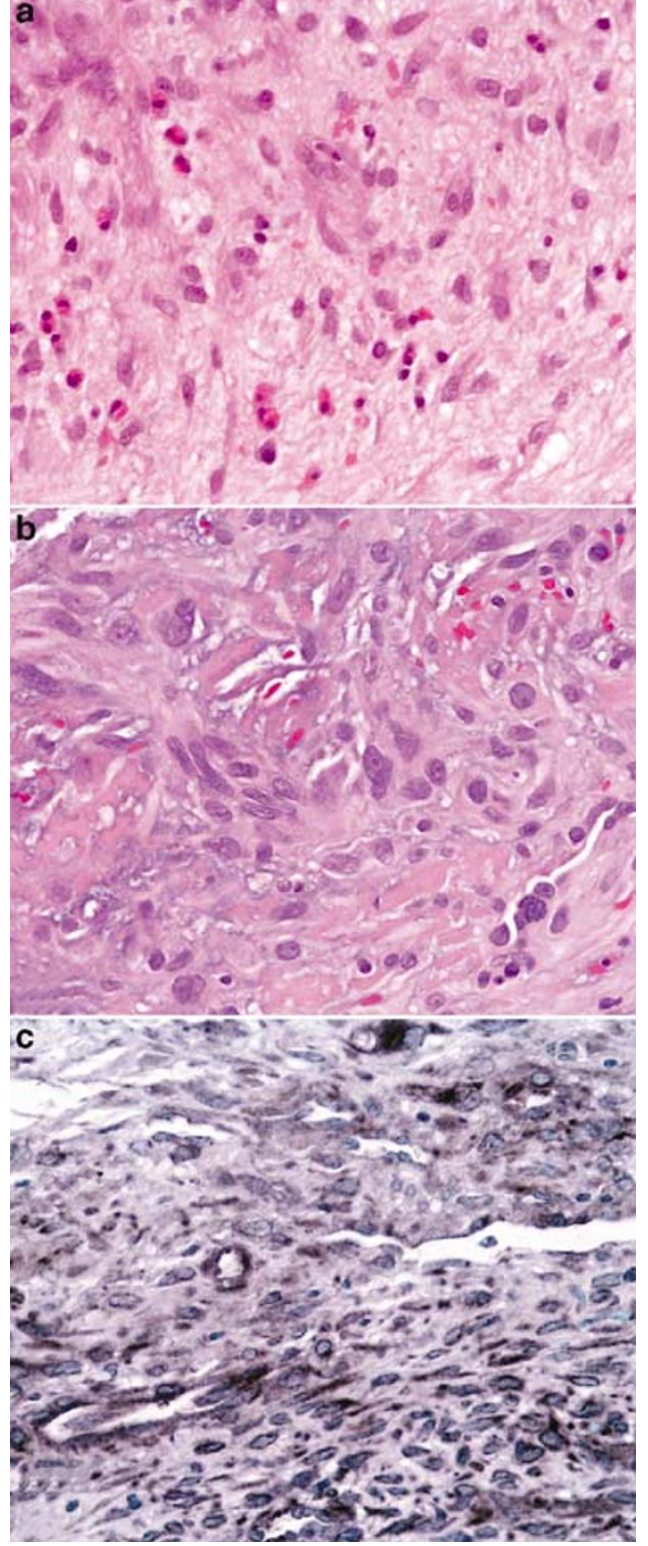

Figure 2 Epithelioid areas, arterial pseudoaneurysms. (a) A sprinkling of eosinophils was not uncommon in epithelioid areas (Case 5). (b) Epithelioid endothelial cells with a rounded to spindled shape (Case 4). (c) Epithelioid cells focally express decorin (Case 18).

region towards the lumen, where the cells were relatively rounded (Figure $7 \mathrm{~b}$ ). Calponin-positive smooth muscle cells were present in every case, but in areas surrounding the proliferating zone, where the smooth muscle cells acquired a more spindled configuration (Figure 7a).

\section{Discussion}

Superficial arterial pseudoaneurysms are most frequent in the face, in the temporal artery or its branches. The anatomic basis for this predilection is
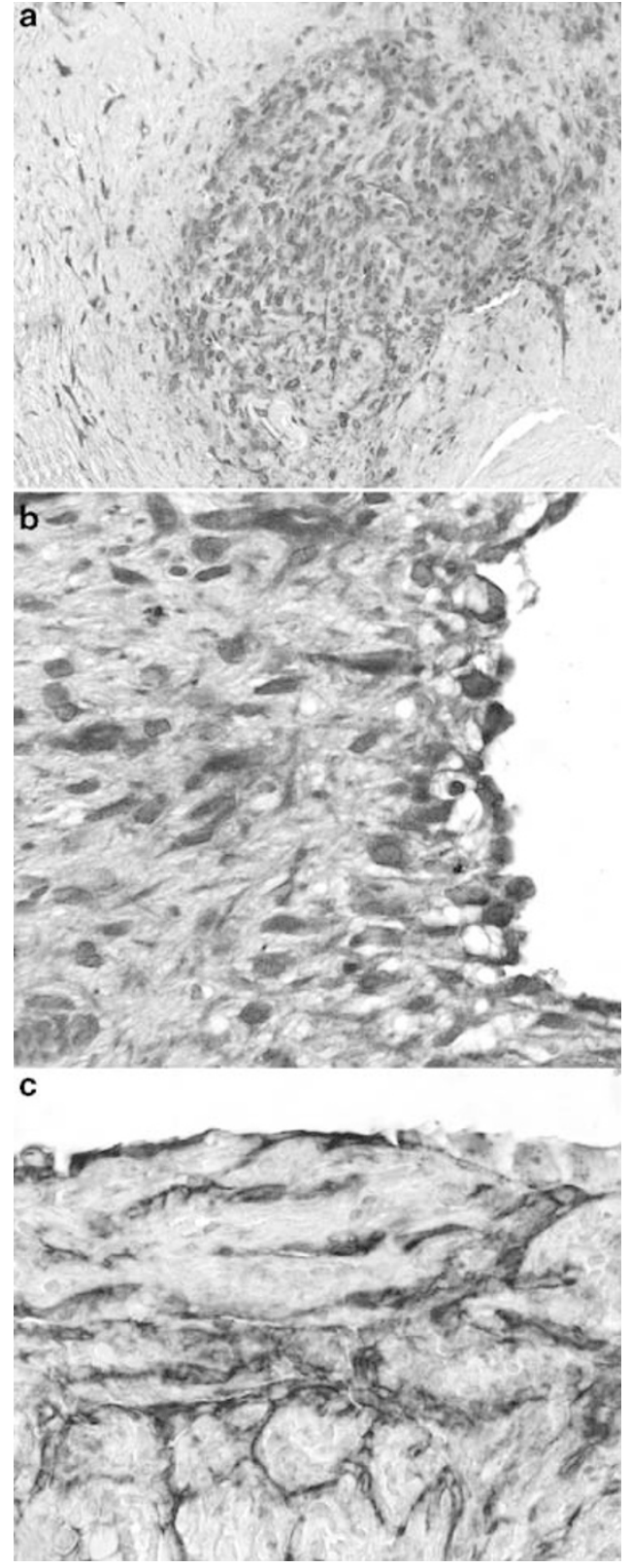

Figure 3 Decorin staining (Case 3). There was a predilection for decorin in areas of neovascularity. (a) Low magnification of granulation tissue area. (b) Near the surface, decorin decorated surface endothelial cells. (c) Endothelial cells express factor VIIIrelated antigen.

related to underlying bony structures and relative lack of soft-tissue support. ${ }^{1}$ It is unknown whether there is an underlying predilection for vascular weakness in patients with pseudoaneurysms. The current study did not support an inherent vasculopathy, as patients with pseudoaneurysms did not have other vascular diseases, and the morphologic appearance of the uninvolved medial remnant was normal. The curious finding of bilaterality in three of 20 temporal aneurysms (15\% of cases) may be secondary to a predisposition to facial trauma, 


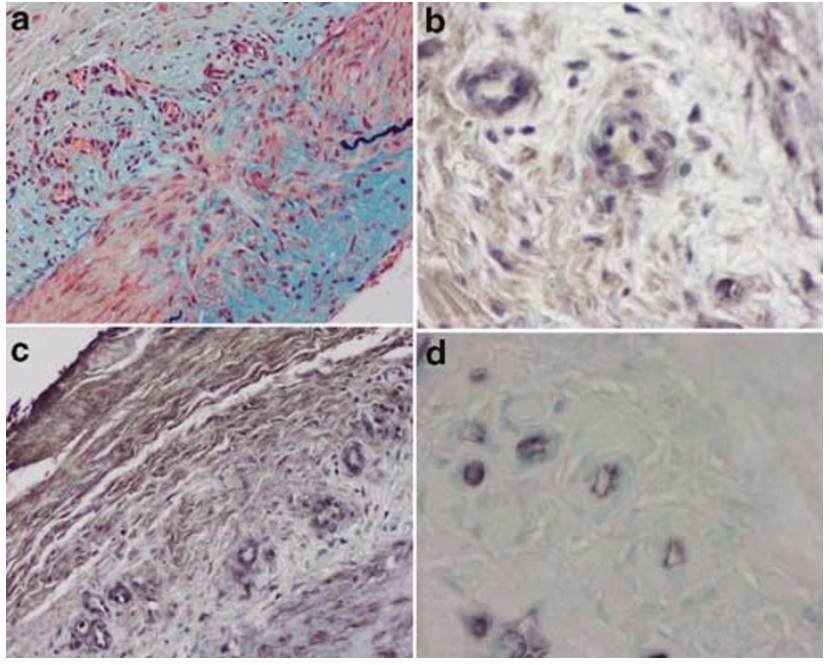

Figure 4 Decorin staining (Case 3). (a) A Movat pentachrome stain shows an interruption of the internal elastic lamina with angiogenesis. ((b)-(d)) There is variable expression of decorin in the endothelial cells of the neovessels.
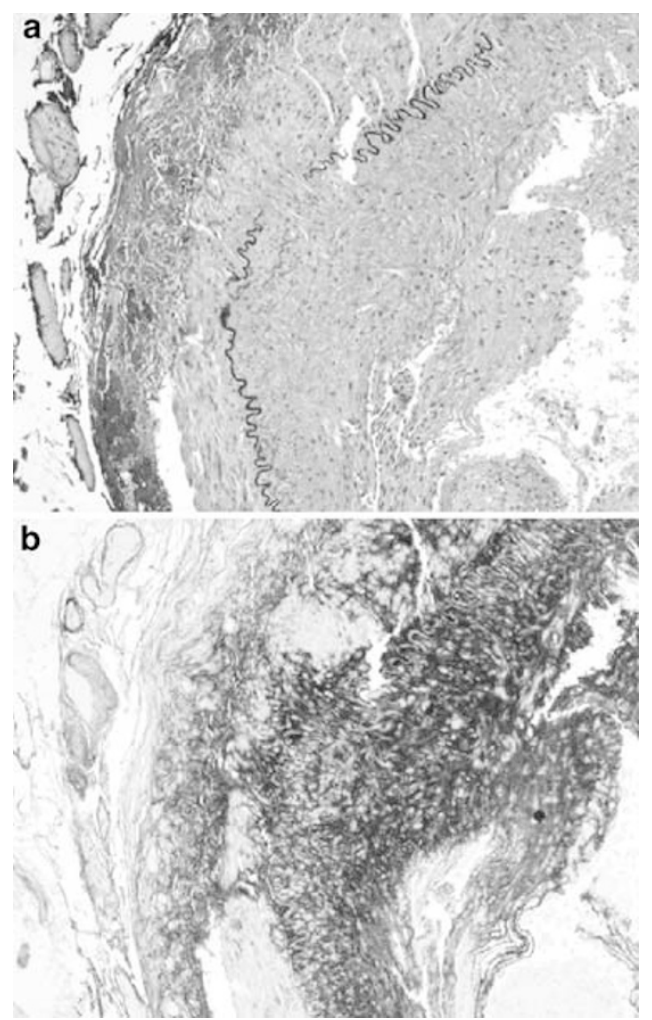

Figure 5 Arterial pseudoaneurysm (Case 5). (a) In this example, the remnant artery with the rupture site was easily identified by elastic stain (Movat pentachrome). (b) Versican demonstrated diffuse staining throughout the myxoid area. The patient was a 16-year-old boy with a protruding temporal mass; he had a history of a similar contralateral lesion.

rather than a congenital or acquired weakness of the temporal arteries.

The unique histologic feature of arterial pseudoaneurysms is the intense deposition of proteogly- a

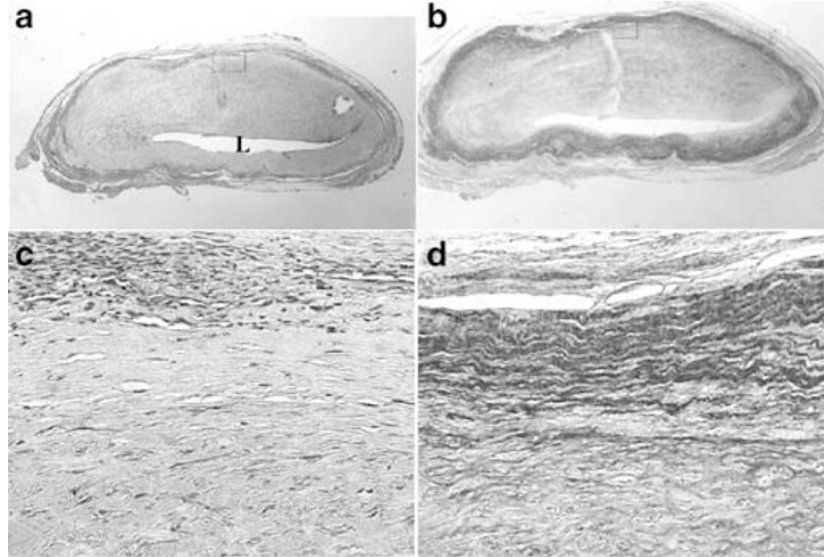

Figure 6 Arterial pseudoaneurysm (Case 23). (a) The lesion at low magnification demonstrates, in this case, a preserved lumen (L) with proteoglycan-rich intimal area. (b) Biglycan shows diffuse peripheral staining. (c) Movat pentachrome shows central area of fibrosis (yellow stain). (d) Similar area of (c) shows biglycan staining in the area of collagen.

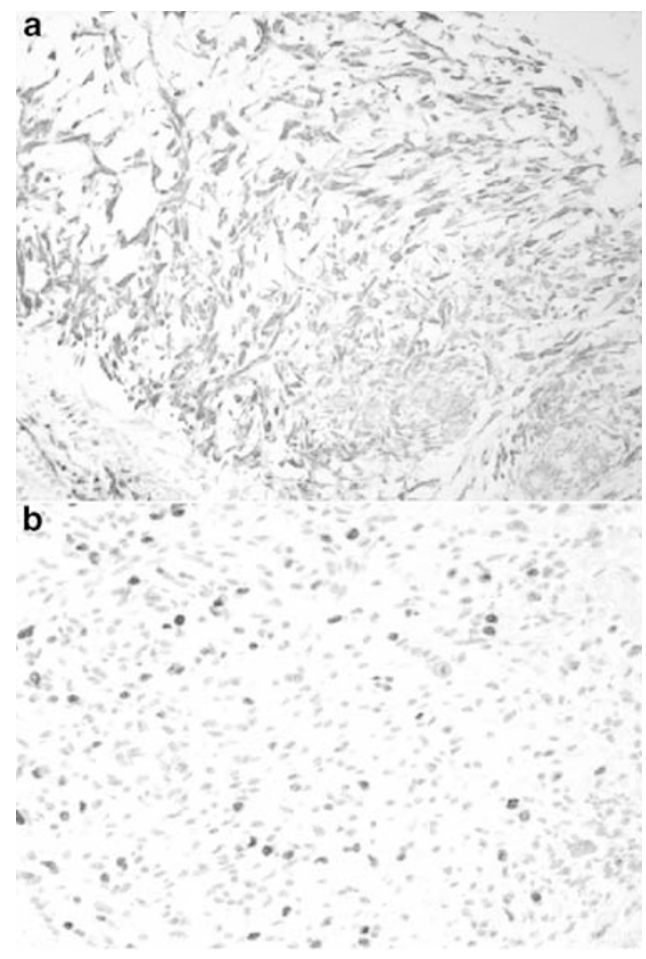

Figure 7 Smooth muscle cell phenotypes. The calponin-positive cells (a) showed elongated cell borders (cytoplasmic stain) and nuclei. The nuclear staining of the proliferating cells with Ki-67 (b) showed more rounded nuclei.

cans in the pseudoaneurysm wall, with marked accumulation of endothelial and smooth muscle cells. This reaction is likely related to the tissue forces caused by the arterial pressures adjacent to the pseudoaneurysm wall. It is interesting that in the single case of known trauma within 2 days, a pseudoaneurysm wall had formed completely 
around the arterial tear, had the highest Ki-67 count, and was composed primarily of endothelial cells, indicating the rapidity of the granulation tissue response. The degree of proteoglycan deposition is far greater than seen in soft-tissue hematomas that form outside the vessel wall. For this reason, the pseudoaneurysm wall is often misinterpreted as a soft-tissue tumor. Alternatively, the inflammation present with the granulation tissue response, together with the exuberant Ki-67-positive smooth muscle cells, may be interpreted as an inflammatory process. In either case, the patient may be unnecessarily evaluated for a neoplasm or vasculitic syndrome. It is imperative that the medial remnant be carefully sought and identified, in order that the appropriate diagnosis is rendered. There is no evidence in patients with excised pseudoaneurysms that anything beyond simple excision is needed.

In the majority of cases examined in the current study, only one rupture site was identified. However, in seven of 23 cases, there were multiple areas of rupture, indicating that the mechanism of medial tear may have been due to compression of the vessel with radial forces causing disruption at multiple sites, instead of penetrating injury caused by a sharp object. This type of injury may explain the lack of a history of trauma in many cases, as blunt trauma may not have resulted in skin lacerations remembered by the patient.

The current study demonstrates a characteristic distribution of proteoglycans within arterial pseudoaneurysms. The large amount of proteoglycans in the granulation tissue response of pseudoaneurysms is likely related to the arterial pressures. Versican is the predominant aggregating proteoglycan (eg, hyaluronan-binding) found in the vasculature. ${ }^{19}$ In the pseudoaneurysms, versican was found in all areas of the granulation tissue response, especially in areas of apparent smooth muscle cell proliferation and migration. In vitro studies show that the pericellular accumulation of versican is required for smooth muscle cell proliferation and migration. ${ }^{20,21}$ Changes in proteoglycan synthesis in response to mechanical strain suggest a structural function in matrix hydration in response to arterial pressures. The nonaggregating proteoglycans, biglycan and decorin, were more selectively found in areas of collagen and endothelial cell proliferation, respectively. In general, the smaller nonaggregating proteoglycans play a limited role in withstanding compression, and interact with other matrix components and contribute to mechanical stability through interaction with collagen. The specific role of nonaggregating proteoglycans in the formation of the pseudoaneurysm wall is unclear, but appears related to collagen deposition and neovascular response.

The presence of decorin-positive microvessels in the areas of the pseudoaneurysms agrees with earlier studies and raises the possibility that decorin is involved in new vessel formation. ${ }^{22}$ Recent studies show that decorin is required for angiogenesis in vitro. ${ }^{23}$ Furthermore, decorin synthesis is upregulated when endothelial cells sprout and form tubes in vitro. ${ }^{24}$ Such studies indicate that specific proteoglycans may regulate specific events that contribute to pseudoaneurysmal disease.

In conclusion, arterial pseudoaneurysms are reactive lesions involving smooth muscle cell proliferation, neovascularity, and marked accumulation of proteoglycans, features that are helpful diagnostically. The distribution of proteoglycans reflected different responses to the vessel injury: versican expression is likely related to tensile stress in the weakened artery, decorin expression is related to neovascularity, and biglycan is expressed in areas of collagen deposition.

\section{Acknowledgements}

The opinions or assertions contained herein are the private views of the authors and are not to be construed as official or reflecting the views of the Department of the Army or the Department of Defense. This work was supported in part by research grants from the National Institutes of Health NIH/NHLBI RO1 HL61799-02, Virmani, and NIH/NHLBI HL 18645-23, Wight.

\section{References}

1 Conner. III WC, Rohrich RJ, Pollock RA. Traumatic aneurysms of the face and temple: a patient report and literature review, 1644 to 1998. Ann Plast Surg 1998;41:321-326.

2 Choo MJ, Yoo IS, Song HK. A traumatic pseudoaneurysm of the superficial temporal artery. Yonsei Med J 1998;39:180-183.

3 Cross WR, Nishikawa H. Traumatic pseudoaneurysm of the superficial temporal artery. J Accid Emerg Med 1999;16:73.

4 Fukuzawa M, Saida T. Pseudoaneurysm of the superficial temporal artery. Acta Derm Venereol 2000;80: 65-66.

5 Han K, Borah GL. Pseudoaneurysm of the anterior superficial temporal artery. Ann Plast Surg 1996;37: 650-653.

6 Manz HJ, Gomes MN. Sports injury as cause of traumatic pseudoaneurysm of superficial temporal artery. Arch Pathol Lab Med 1984;108:775-776.

7 Merkus JW, Nieuwenhuijzen GA, Jacobs PP, et al. Traumatic pseudoaneurysm of the superficial temporal artery. Injury 1994;25:468-471.

8 Nemade SS, Eiman M, Blondet R, et al. Pseudoaneurysm of the superficial temporal artery. South Med J 1996;89:815-817.

9 Pennington C, Lewis JV. Traumatic pseudoaneurysm of the superficial temporal artery. Tenn Med 1997;90:284.

10 Webber CM, Wind GG, Burton RG. Pseudoaneurysm of the superficial temporal artery: report of a case. J Oral Maxillofac Surg 1997;55:166-169.

11 Weller CB, Reeder C. Traumatic pseudoaneurysm of the superficial temporal artery: two cases. J Am Osteopath Assoc 2001;101:284-287. 
12 Suzuki Y, Sasaki Y, Shimizu H. Non-pulsatile aneurysm of the superficial temporal artery. Br J Dermatol 1999;140:781-782.

13 Evanko S, Raines EW, Ross R, et al. Proteoglycan distribution in lesions of atherosclerosis depends on lesion severity, structural characteristics and the proximity of PDGF and TGF-beta1. Am J Pathol 1998;152:533-546.

14 Wight TN, Lara S, Reissen R, et al. Selective deposits of versican in the extracellular matrix of restenotic lesions from human peripheral arteries. Am J Pathol 1997;151:963-973.

15 Gutierrez PS, O’Brien KD, Ferguson M, et al. Differences in the distribution of versican, decorin, and biglycan in atherosclerotic human coronary arteries. Cardiovasc Pathol 1997;6:271-278.

16 Evanko SP, Vogel KG. Proteoglycan synthesis in fetal tendon is differentially regulated by cyclic compression in vitro. Arch Biochem Biophys 1993;307: 153-164.

17 Lee RT, Yamamoto C, Feng Y, et al. Mechanical strain induces specific changes in the synthesis and organization of proteoglycans by vascular smooth muscle cells. J Biol Chem 2001;276:13847-13851.

18 Lara SL, Evanko SP, Wight TN. Morphological evaluation of proteoglycans in cells and tissues. Methods Mol Biol 2001;171:271-290.
19 Yao LY, Moody C, Schönherr E, et al. Identification of the proteoglycan versican in aorta and smooth muscle cells by DNA sequence analysis, in situ hybridization and immunohistochemistry. Matrix Biol 1994;4:213-225.

20 Evanko SP, Johnson PY, Braun KR, et al. Plateletderived growth factor stimulates the formation of versican-hyaluronan aggregates and pericellular matrix expansion in arterial smooth muscle cells. Arch Biochem Biophys 2001;394:29-38.

21 Evanko SP, Angello JC, Wight TN. Formation of hyaluronan and versican-rich pericellular matrix is required for proliferation and migration of vascular smooth muscle cells. Arterioscler Thromb Vasc Biol 1999;19:1004-1013.

22 Nelimarkka L, Salminen H, Kuopio T, et al. Decorin is produced by capillary endothelial cells in inflammation-associated angiogenesis. Am J Pathol 2001;158: 345-353.

23 Schönherr E, O’Connell BC, Schittny J, et al. Paracrine or virus-mediated induction of decorin expression by endothelial cells contributes to tube formation and prevention of apoptosis in collagen lattices. Eur J Cell Biol 1999;78:44-55.

24 Järvelainen HT, Iruela-Arispe ML, Kinsella MG, et al. Expression of decorin by sprouting bovine aortic endothelial cells exhibiting angiogenesis in vitro. Exp Cell Res 1992;203:395-401. 